"This is a pre-print of an article published in European Polymer Journal. The final authenticated version is available online at: https://doi.org/10.1016/j.eurpolymj.2018.06.026”.

"Esta es una preimpresión de un artículo publicado en European Polymer Journal. La versión final autenticada está disponible en línea en: https://doi.org/10.1016/j.eurpolymj.2018.06.026 ".

\title{
Spatial control and cell adhesion selectivity on model gold surfaces grafted with elastin- like recombinamers
}

Tatjana Flora ${ }^{1}$, I. González de Torre ${ }^{2}$, Luis Quintanilla ${ }^{1}$, M. Alonso ${ }^{1}$, J. Carlos Rodríguez-Cabello ${ }^{1}$

${ }^{1}$ BIOFORGE, CIBER-BBN, Edificio Lucia, Universidad de Valladolid, Paseo Belén 19, 47011, Valladolid, Spain

${ }^{2}$ TECHNICAL PROTEINS NANOBIOTECHNOLOGY (TPNBT S.L.) Paseo Belén 9 A, 47011, Valladolid, Spain

Tatjana Flora

G.I.R BIOFORGE, Universidad de Valladolid

Paseo de Belén 19

47011, Valladolid, Spain

E-mail: tflora@bioforge.uva.es

Dr. Israel Gonzalez de Torre

TECHNICAL PROTEINS NANOBIOTECHNOLOGY (TPNBT S.L.)

Paseo Belén $9 \mathrm{~A}$

47011, Valladolid, Spain.

E-mail: igonzalez@tpnbt.com

Prof. Luis Quintanilla

G.I.R BIOFORGE, Universidad de Valladolid

Paseo de Belén 19

47011, Valladolid, Spain

E-mail: luisq@ele.uva.es

Prof. Matilde Alonso

G.I.R BIOFORGE, Universidad de Valladolid

Paseo de Belén 19

47011, Valladolid, Spain

E-mail: malonso@bioforge.uva.es

Prof. José Carlos Rodríguez Cabello

G.I.R BIOFORGE, Universidad de Valladolid

Paseo de Belén 19

47011, Valladolid, Spain

E-mail: roca@bioforge.uva.es 


\begin{abstract}
A simple system for cell selectivity and spatially controlled cell adhesion has been developed using model gold surfaces grafted with a combination of two ELRs containing into their backbone celladhesion domains such as RGD and REDV. Grafting onto gold was achieved via redox reaction through thiol groups present in amino terminal cysteine tails of the ELRs. The correlation among contact angle, SEM micrographs, AFM, XPS and QCM-D have been carried out.

After in-depth adhesion studies, a mixture of 75\% ELR-REDV and 25\% ELR-RGD was found to exhibit high selectivity for endothelial cells, promoting strong adhesion thereof.

Consequently, certain areas of gold surfaces (strips) were cleaned by laser ablation and functionalized with the mixture 75\% ELR-REDV - 25\% ELR-RGD leading to a spatial segregation of the co-culture made of HUVEC and HFF1 cells. This platform therefore exhibits selective spatial control over cell adhesion associated with the bioactive epitopes (RGD and REDV) contained in the ELR sequence, since each functionalized surface (including strips) have similar topographic, hydrophobic and mechanical properties.
\end{abstract}

Keywords: surface bio-functionalization; elastin-like recombinamers; selectivity; laser ablation; spatial control. 


\section{Introduction}

Control of cell performance such as spatial control and selectivity plays an important role in generation of a favorable environment to develop interfaces for further applications in various research fields such as microelectronics and biomedicine.[1-3] Bio-functionalization of surfaces with cell adhesion molecules has attracted significant interest that lead to the achievement of systems with bespoke properties such as wettability, adhesion, and biocompatibility namely to a thorough control over cellular response. [4-8] The most widely used methods for functionalization are difficult to control as they have several drawbacks essentially uncontrolled protein bonding, changes of the protein bioactivity and its denaturation.[9, 10] Thus, it is mandatory to obtain information about the structure of the proteins, their interaction with the support surface and the interaction of the proteins with other proteins. Additionally, surface chemistry and topography are important parameters that may influence protein adsorption, cell interaction, and lastly the host response. [11]

Currently, recombinant proteins such as elastin-like recombinamers (ELRs) can overcome several drawbacks such as immunological aspects and moreover can enhance their biological function and control cell-material interaction.[12-14] ELRs are based on the repetition of certain sequences from natural elastin, specifically the pentapeptide (VPGXG), where X can stand for any amino acid except L-proline. [15] [16] [17] This sequence confers elastic properties on the resulting recombinamer along with many other of their most striking properties, such as high biocompatibility and lower critical solution temperature behavior. $[15,18]$ ELRs are produced by recombinant techniques, thus allowing total control over the amino-acid sequence and a completely tailored design of the polypeptide chain at the gene level.[19-21]

The majority of extracellular matrix molecules contain a range of structural domains with specific functions. [22] REDV (arginine-glutamic acid-aspartic acid-valine) is a specific adhesive sequence for endothelial cells found in the alternatively-spliced IIICS-5 domain of human plasma fibronectin. This motif mediate cell adhesion and spreading via $\alpha_{4} \beta_{1}$ integrin in endothelial cells, but not in smooth muscle cells or fibroblasts. [23] [24] On the other hand, RGD is a universal cell adhesive sequence within fibronectin that mediates cell attachment, and present in numerous proteins such as integrins. A subset of integrins recognize the RGD motif within their ligands, the binding of which mediates both 
cell-substrate and cell-cell interactions. [25] Due to the previously mentioned recombinant nature of ELRs, they allow the inclusion of bioactive domains such as REDV or RGD promoting in this way cell adhesion and cell spreading via its receptor. Both REDV and RGD have been considered in this work, thus leading to the selective adhesion of endothelial cells due to their high specificity. Moreover, amino terminal cysteines (Cys, C-block) have been incorporated into the ELRs employed in this work (CC-RGD and CCC-REDV) in order to allow grafting to gold surfaces via a redox reaction. [26] There are numerous characteristics of gold that make it a good choice as a substrate to study biological events. Firstly, gold is an inert metal, easy to obtain, compatible with cells, namely cells can adhere and proliferate without evidence of toxicity and lastly is easy to pattern by a combination of lithographic tools. [27, 28] Initially, the mostly used technique to obtain patterns was photolithography which was substitute by soft lithography, direct writing and laser ablation.[29-31] Laser ablation technique generate a high precision surface topography for the self-organized and direct fabrication of nano- and microstructures on a surface. [32, 33] This technique has many advantages over other methods such as low surface contamination, low mechanical damage, and controllable surface structuring of three-dimensional components.[34] [35] This technique is characterized by a great recent progress due to its ability to control not only physical, but also chemical surface features and will continue to be developed into a more standardized research tool in biology. Selective laser ablation is applied where individual layers have to be ablated without significantly damaging the layers or substrates below leaving the surfaces of conducting core material undamaged and without process marks such as cracks like at minimal heat input. A prominent analytical technique, namely matrix assisted laser desorption/ionization (MALDI), provides a means of ejecting intact molecules—even large biomolecules—into the gas phase. [36] The kinetic energies of ablated particles are typically high enough to promote surface diffusion, but not so high as to induce bulk damage. [37] Ultrashort pulse laser ablation is very beneficial given the large variety of structures that can be fabricated, the limited number of heat-affected zones that cannot be transferred to the surrounding material, and its high precision and reproducibility. [38] These findings suggest the possibility of producing biomaterial interfaces for electronic devices and widespread use in the majority of biological applications. [39] 
This work describes the development of an efficient system in which the selective adhesion of a coculture made of HUVECs and HFF-1 cells is spatially controlled due to the use of two ELRs that have a complementary bioactivity (CC-RGD and CCC-REDV) and include cysteines at the amino terminal region to promote a covalent linkage to gold surfaces.[40] We will demonstrate that an optimal composition of 75\% CCC-REDV and 25\% CC-RGD leads to the selective adhesion of endothelial cells in the co-culture system. To achieve this goal, whole model gold surfaces (Fig. 1a) were initially bio-functionalized with the ELR including CC-RGD (Fig. 1b). Strips cleaned by laser ablation (Fig. 1c), were afterward grafted with a previously optimized proportion of a mixture of two ELRs (25\%CC-RGD and 75\% CCC-REDV) (Fig. 1d). Finally, a co-culture of cells was employed and spatially controlled cell adhesion was obtained. (Fig. 1 e-f). The synergy between two different bioactivities, which produces an enhanced cell-selectivity effect, is clearly demonstrated.

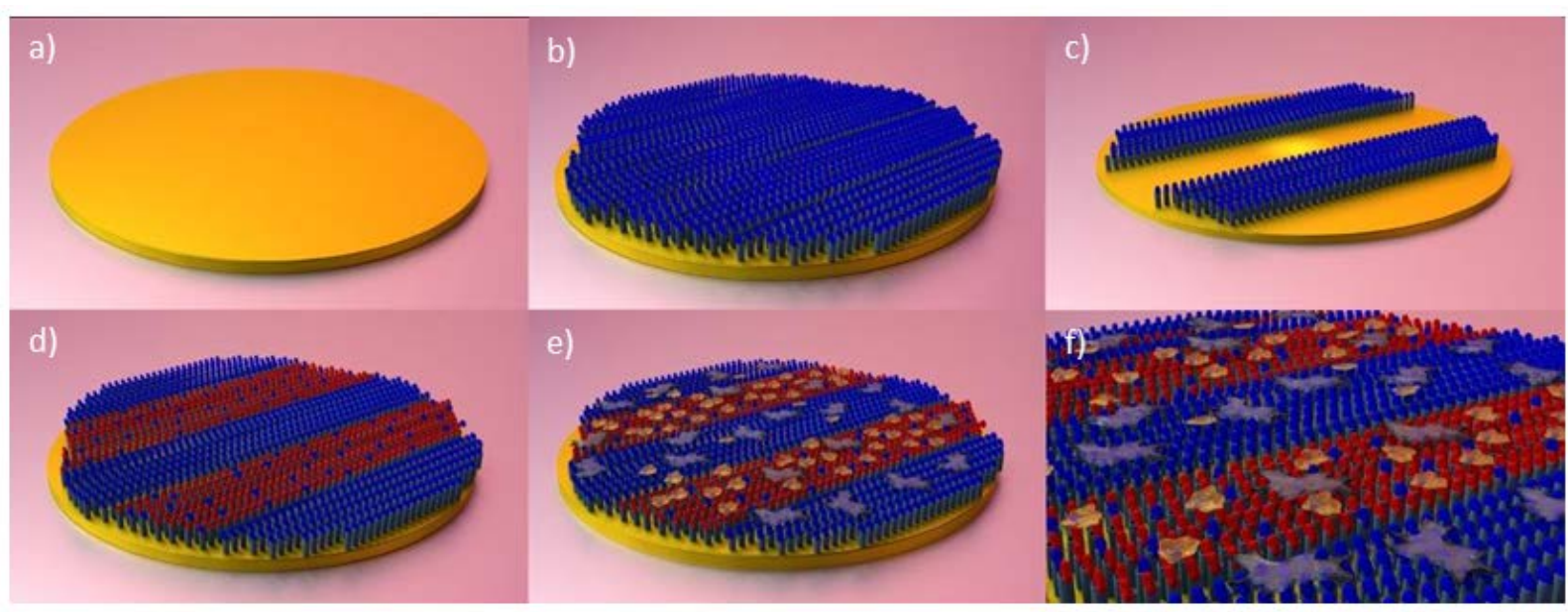


I)

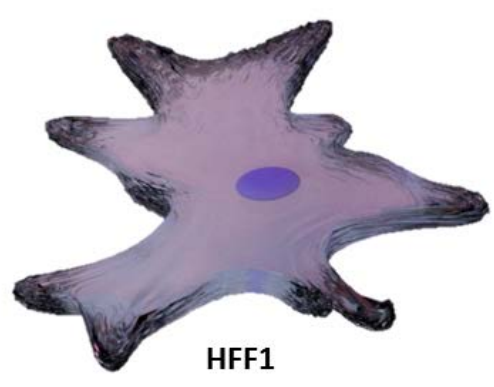

II)

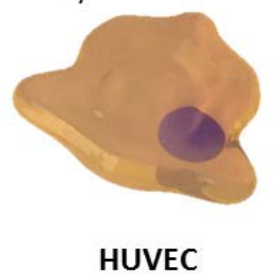

III)

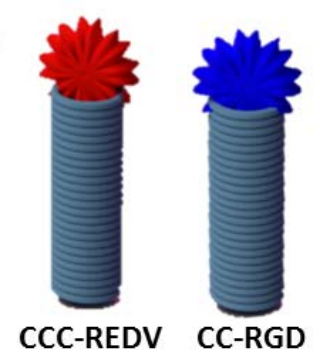

Figure 1: Schematic representation of the bio-functionalized surface: a) non-functionalized gold surface; b) gold surface functionalized with CC-RGD recombinamer; c) gold surface ablated with MALDI TOF/TOF laser; d) ablated strips functionalized with 75\% ELR-REDV/25\% ELR-RGD; e) adhesion of HUVEC and HFF1 cells; f) magnification of adhered cells.

I) fibroblast cells; II) endothelial cells; III) ELR-REDV (red color), and ELR-RGD (blue color) recombinamers beyond the transition temperature. 


\section{Materials and Methods}

\subsection{Materials:}

2.1.1 Gold surfaces (glass coverslips with a thickness of 0.13 to $0.16 \mathrm{~mm}$ were coated with a gold layer) were purchased from AMSBIO Company (Madrid, Spain). A diameter of $1 \mathrm{~cm}$ was coated with gold with a thickness of $10 \mathrm{~nm}$. The thin gold film was deposited onto a supporting substrate by sputtering (the material to be deposited is ejected from a source by plasma discharge and then deposited onto the substrates).

2.1.2 Elastin-Like Recombinamers: The ELRs were constructed using standard genetic engineering techniques. [15] The bio-produced recombinamers were purified by a series of centrifugations under and above their transition temperature $\left(\mathrm{T}_{\mathrm{t}}\right)$ and were dialyzed against ultrapure water (MilliQ) before being lyophilized. Three different ELRs containing the cysteines required for surface immobilization at the amino terminal region were produced. The first one (CC-RGD) contains a cell-adhesive RGD (Arg-Gly-Asp) sequence and includes two C-blocks; the second one (CCC-REDV) contains REDV (Arg-Glu-Asp-Val) and includes three C-blocks, and the last one (CCC-EI) was synthesized as a control polymer in cell co-cultures with no bioactive sequence, which showed antifouling behavior. The sequences of the three ELRs used in this work are:

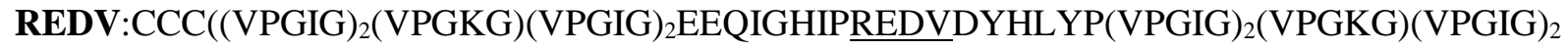
)$_{10}$

RGD:CC((VPGIG) $)_{2}(\mathrm{VPGKG})(\mathrm{VPGIG})_{2}$ EEQIGHIPRGDDYHLYP(VPGIG) ${ }_{2}(\mathrm{VPGKG})(\mathrm{VPGIG})_{2}(\mathrm{VG}$ VAPG) $\left.)_{3}\right)_{10}$

\section{EI:CCC[(VPGVG) $)_{2}$ VPGEG(VPGVG) $\left.{ }_{2}\right]_{10}(\mathrm{VGIPG})_{60} \mathrm{~V}$}

The purity and molecular weight of the ELRs were verified by sodium dodecyl sulfatepolyacrylamide gel electrophoresis and matrix-assisted laser desorption/ionization time-of-flight (MALDI-TOF) mass spectrometry. An amino acid composition analysis was also performed. Additional characterization of the ELRs was accomplished by infrared spectroscopy, differential scanning calorimetry (DSC) and nuclear magnetic resonance (NMR) techniques. The characterization 
results for the ELRs are provided as Supporting Information (see Figs. S1-S5 in Supporting Information). The corresponding ELR transition temperatures are: $31{ }^{\circ} \mathrm{C}$ (CC-RGD), $29{ }^{\circ} \mathrm{C}$ (CCCREDV), and $30^{\circ} \mathrm{C}$ (CCC-EI) (see Fig. S2 in Supporting Information).

2.1.3 Cell culture: Human umbilical vein endothelial cells (HUVEC) were purchased from Life Technologies S.A. (Madrid, Spain). These cells were cultured at $37{ }^{\circ} \mathrm{C}$ and $5 \% \mathrm{CO}_{2}$ in $\mathrm{MED200}$ culture medium (M200500, Life Technologies S.A, Madrid, Spain) supplemented with 100 U/100 mg/mL penicillin/streptomycin (15140-122, Invitrogen Corporation, Madrid, Spain). HUVEC between passages 2 and 6 were used in all experiments. Human foreskin fibroblasts (HFF1) were purchased from Life Technologies S.A. (Madrid, Spain) and were cultured in DMEM medium supplemented with $15 \% \mathrm{FBS}$ and $100 \mathrm{U} / 100 \mathrm{mg} / \mathrm{mL}$ penicillin/streptomycin at $37{ }^{\circ} \mathrm{C}$ and $10 \% \mathrm{CO}_{2}$. Fibroblasts between passages 6 and 10 were used in all experiments. The cells were labeled with PKH67 (green fluorescent dye) and PKH26 (red fluorescent dye) respectively. PKH67 and PKH26 were purchased from Sigma-Aldrich Quimica S.L. (Madrid, Spain). The bio-functionalized gold surfaces were placed in a 24-well culture plate (Nunc $^{\mathrm{TM}}$ Cell-Culture Treated Multidishes, Life Technologies, Madrid, Spain).

\subsection{Methods:}

\subsubsection{Surface cleaning/activation:}

The surfaces were treated with Plasma Cleaner (Sterilizer PDC-002, Harrick Scientific Corporation, USA) using Argon plasma for 20 minutes at a high power setting (30 $\mathrm{W}$ applied to the RF coil) in order to etch the surface before each experiment.

\subsubsection{Surface functionalization}

After cleaning/activation by plasma treatment, the surfaces were immersed in ELR solutions with a concentration of $5 \mathrm{mg} / \mathrm{mL}$ for 6 hours. Although several ELR concentrations were considered, a value $5 \mathrm{mg} / \mathrm{mL}$ was selected due to the homogeneous coverage obtained analyzed by SEM. Subsequently, several washing steps with milliQ water were performed in order to remove remnants of ELRs not 
grafted to the surfaces. Finally, the surfaces were dried under a nitrogen flow and submitted to several treatments reported below.

\subsubsection{Contact Angle}

Contact angle measurements were performed using the sessile drop method with a Data Physics OCA20 system instrument. The drop profile images during micro-syringe dispensation were recorded using an adapted CCD video camera. The stainless-steel needle tip was always kept at the top of the sessile drop and immersion of the needle into the drop was avoided during the measurements to prevent distortion of the drop shape by the needle. Ten measurements from different locations on each surface were measured to ensure a representative value of the contact angle.

\subsubsection{X-ray Photoelectron Spectroscopy (XPS):}

XPS experiments were carried out using a Physical Electronics (PHI) 5500 spectrometer equipped with a monochromatic X-ray source (Al Ka line with an energy of $1486.6 \mathrm{eV}$ and $350 \mathrm{~W}$ ). The pressure inside the analysis chamber was $10^{-7} \mathrm{~Pa}$. All measurements were performed at an angle of $45^{\circ}$ with respect to both the X-ray source and analyzer. Survey scans were taken in the range 0-1100 eV and high resolution scans were obtained for the $\mathrm{C}_{1 \mathrm{~s}}, \mathrm{~N}_{1 \mathrm{~s}}, \mathrm{O}_{1 \mathrm{~s}}$ and Au peaks. The elemental surface composition was estimated from the area of the different photoemission peaks taken from the survey scans modified by their corresponding sensitivity factors.

\subsubsection{Scanning Electron Microscopy (SEM):}

Scanning electron microscopy (SEM) was used to investigate the surface morphology. Thus, the surfaces were immersed in recombinamer solutions with a concentration of $5 \mathrm{mg} / \mathrm{mL}$ for 6 hours. After several washing steps with MilliQ water, the surfaces were dried with gaseous nitrogen. Micrographs were obtained using a scanning electron microscope (FEI Quanta 200 FEG) in low vacuum mode at 3 keV. Morphological details were evaluated quantitatively using the ZEN (Blue Edition, 2012) software package (Carl Zeiss Microscopy). 


\subsubsection{Atomic Force Microscopy (AFM)}

AFM measurements were performed using a Dimension 3100 microscope controlled by a Nanoscope IV controller system (Digital Instruments) in tapping mode using $\mathrm{V}$-shaped $\mathrm{Si}_{3} \mathrm{~N}_{4}$ tips (OMCL TR400PSA, Olympus, Japan). AFM images were taken from PBS immersed samples, with a nominal spring constant of $0.32 \mathrm{~N} / \mathrm{m}$. The scan area was $10 \mu \mathrm{m} \times 10 \mu \mathrm{m}$ and the root-mean-square (RMS) roughness of the surfaces was evaluated for regions of $1 \mu \mathrm{m} \times 1 \mu \mathrm{m}$. Surface roughness quantifies the vertical variations of a real surface from its ideal form. One of the most common parameters used to describe the degree of roughness of a surface is the root mean square (RMS) roughness, which is defined as (Equation 1):

$$
R q=\sqrt{\frac{1}{n}}\left(\sum_{i=1}^{n} y_{i}^{2}\right) \quad \text { (Equation 1) }
$$

where "n" corresponds to the total number of pixels in the image and "i" corresponds to the number of individual pixels. This parameter is especially useful when these variations in Z height are positive and negative from the ideal mean line. Five randomized sections were created for each image to determine the surface roughness of the polymeric surfaces.

\subsubsection{Quartz Crystal microbalance with Dissipation}

The QCM-D technique was applied in order to estimate the physical parameters representing the ELR thin film adsorbed on the surface. [45-46] This technique allows simultaneous measurements of both frequency and energy dissipation changes.

A Q-Sense Explorer System equipment (Biolin Scientific, Sweden) was used, with a peristaltic pump pumping every solution through the circular flow-through circuit at a flow rate of $50 \mu \mathrm{L} / \mathrm{min}$.An ATcut $5 \mathrm{MHz}$ quartz crystal coated with gold (Biolin Scientific) was used as sensor, and the frequency and dissipation changes were recorded up to the 13th overtone number. The following sequence of flows (or events) was allowed to occur in each QCM-D measurement. First, since PBS was used as buffer for the polymer solutions, a flow of PBS was injected for 3 minutes to define a stable baseline, followed by the flow of the polymer solution under study for 20 minutes; the last event corresponded to a buffer rinse (10 minutes). Measurements in which flow of the polymer solution (RGD and 75\% 
REDV/25\% RGD) was maintained for 6 hours (corresponding to the time used in the surface functionalization for cell adhesion studies) were also performed. In this case, the PBS rinse lasted overnight. All measurements were carried out at $23^{\circ} \mathrm{C}$ and 3 replicates were performed for each.

The QCM-D experimental data were numerically fitted to the Voight (continuous) viscoelastic model [52-53] using Dantzig’s Simplex algorithm as implemented in the software from Biolin Scientific (QSense Dfind). An explicit consideration of the frequency dependence of viscoelastic properties was assumed according to a power law. A descending incremental fitting was used, with the quality of the fitting being determined by the parameter $\chi^{2}$ (lower $\chi^{2}$ values indicate a better fitting). Only fits providing fitting without jumps between solutions in the resulting physical parameters have been considered.

\subsubsection{Laser ablation}

After functionalization of the whole surface with 100\% CC-RGD, some regions (width: $200 \mu \mathrm{m}$ ) were ablated using a smart beam MALDI laser in a MALDI TOF/TOF UltrafleXtrem instrument from Bruker (Center for Omic Sciences, Reus, Spain). Smartbeam technology combines the speed of a solid-state laser with the wide range of applications associated with nitrogen lasers. The new UltrafleXtreme, with a $1000 \mathrm{~Hz}$ smartbeam-II laser, enables laser focus diameters down to $10 \mu \mathrm{m}$ for high spatial resolution imaging without pixel overlap. The Smartbeam MALDI laser was used at low intensity by preparing 500 shots/raster spots at $20 \%$ laser intensity and a large smartbeam.

\subsubsection{In vitro studies}

A reference standard curve was created to convert sample fluorescence values into cell numbers per unit well area. HUVEC and HFF1 cells were labeled following the protocols for the PKH67 Fluorescent Cell Linker Kit and PKH26 Red Fluorescent Cell Linker Kit, respectively. A range from 500 to 4000 cells was used. The plates were incubated for 4 hours at $37^{\circ} \mathrm{C}$ in a $5 \% \mathrm{CO}_{2}$ atmosphere with the corresponding medium to allow attachment of the cells. A fluorescence microplate reader with appropriate filters (490 nm excitation, and $502 \mathrm{~nm}$ emission for green fluorescence; $551 \mathrm{~nm}$ excitation, and $567 \mathrm{~nm}$ emission for red fluorescence) was used to measure the sample fluorescence. 
Cells were seeded onto the surfaces at a density of $5 \cdot 10^{3}$ cells/well using MED200 culture medium supplemented with $100 \mathrm{U} / 100 \mathrm{mg} / \mathrm{mL}$ penicillin/streptomycin. After culture, the culture medium was removed and the gold surfaces were washed with DPBS (Dulbecco's Phosphate-Buffered Saline, Life Technologies, Madrid, Spain). Adhesion was evaluated by measuring the fluorescence with a photometer plate reader. Fluorescence images were also used to count the HUVEC and HFF1 cells adhered to the strips using Fiji software.

\subsubsection{Statistical Analysis}

Data are reported as mean \pm SD $(n=3)$. Statistical analysis was evaluated by one-way analysis of variance using the Holm-Sidak method. A $p$ value of less than 0.05 was considered to be statistically significant. $\left({ }^{* *}\right) p<0.001,\left({ }^{*}\right) p<0.05$, and $p>0.05$ indicates no significant differences (n.s.d.). 


\section{Results}

\subsection{Contact Angle}

The functionalization of gold surfaces were characterized by contact angle measurements (Fig. 2). Thus, non-functionalized and non-activated (control) surfaces exhibited average contact angles of $57^{\circ}$, whereas after functionalization these values increased to around $92^{\circ}$. The contact angle for activated control surfaces was small enough to be measured under our experimental conditions $\left(<8^{\circ}\right)$. As expected, the contact angle was significantly affected by functionalization, with the high value of the contact angle corresponding to a high surface hydrophobicity. No significant differences were found for the three samples functionalized with ELRs. Even after vigorous rinsing with MilliQ water, a layer of recombinamers remains grafted to the surface.

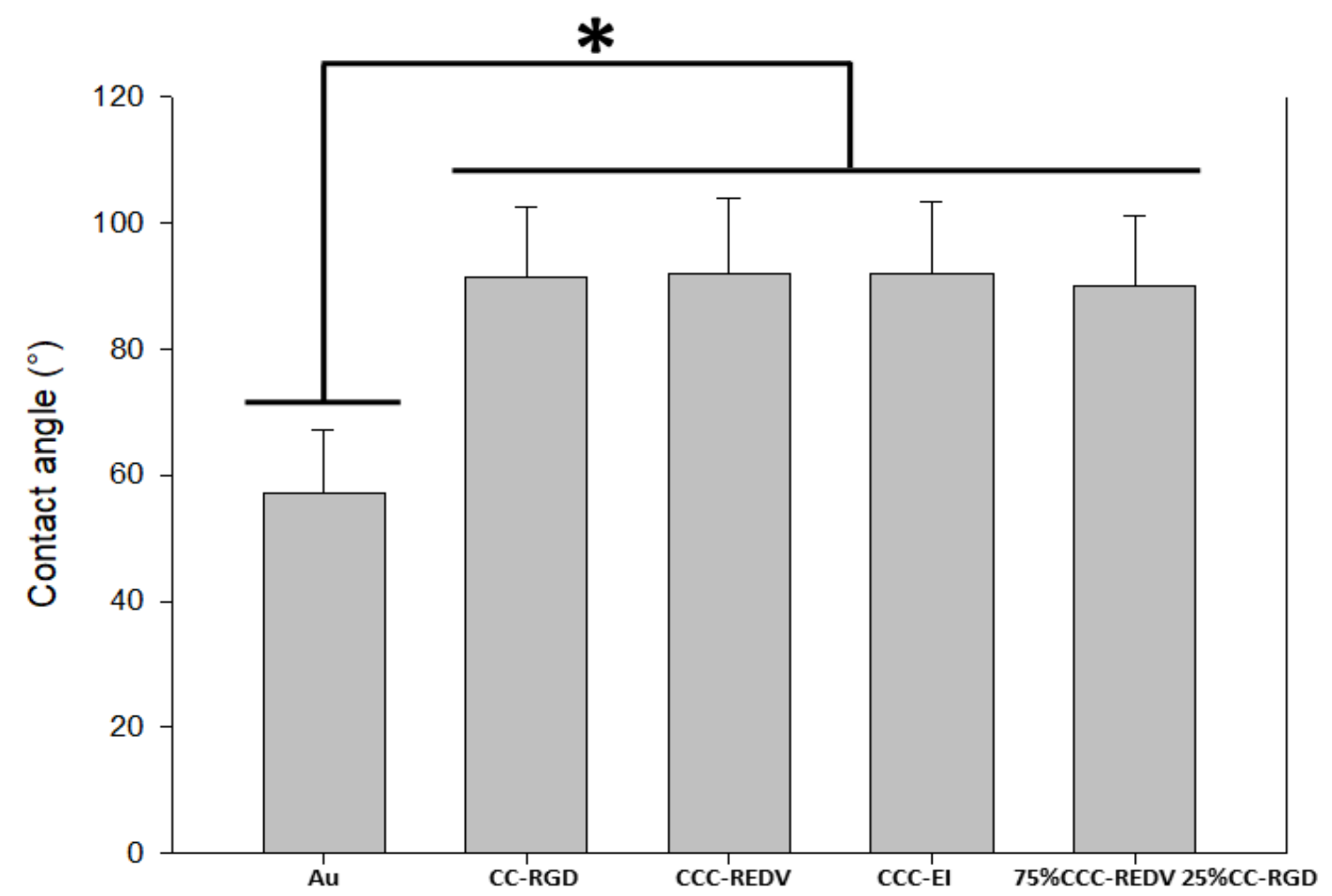

Figure 2: Contact angle for a non-functionalized and non-activated surface (Au), surface functionalized with CC-RGD, surface functionalized with CCC-REDV and surface functionalized with CCC-EI. Data are reported as mean \pm SD $(n=3)$. Statistical analysis was evaluated by analysis of variance using the Holm-Sidak method. ${ }^{*} p<0.05$; n.s.d., no significant differences. 


\subsection{X-Ray Photoelectron Microscopy (XPS)}

XPS is the most widely used surface-analysis technique as it can be applied to a broad range of materials and provides valuable quantitative and chemical information about the surface of the material being studied. Detailed XPS spectra of some fundamental elements can be found in the Supporting Information (Fig. S6). Table 1 shows the atomic percentage for some significant surface atoms for the functionalized surfaces, control sample and the strip ablated and subsequently functionalized with 75\% CCC-REDV/25\% CC-RGD. The orbitals used to calculate concentrations were C1s, O1s, N1s, Au4f and Au4d. The atomic ratios of the most significant elements on the gold surfaces $(\mathrm{C}, \mathrm{O}, \mathrm{N}$, and $\mathrm{Au})$ were examined. Thus, whereas a marked variation in the percentage of the most representative atoms for each surface with respect to the control Au surface was observed, no significant differences are observed between the different functionalized surfaces and the strip. The Au4f percentage decreases and the proportion of carbon and nitrogen (typical atoms in ELRs) increases. The high level of carbon is mainly due to extrinsic hydrocarbon deposition on the surfaces since these samples were exposed to air prior to the XPS analysis.

Table 1. Atomic percentages for $\mathrm{C}, \mathrm{O}, \mathrm{N}$ and $\mathrm{Au} 4 \mathrm{f}$ in control (Au) and functionalized surfaces.

\begin{tabular}{llllc} 
& C1s (\%) & N1s (\%) & O1s (\%) & Au4f (\%) \\
\hline Au (control) & 30.11 & 0.99 & 12.31 & 56.59 \\
\hline CCC-REDV & 57.77 & 12.33 & 14.52 & 15.39 \\
CC-RGD & 51 & 9.4 & 30 & 14.22 \\
CCC-EI & 59.82 & 15.77 & 15.14 & 9.27 \\
STRIP (75\%CCC-REDV-25\% CC-RGD) & 66.01 & 13.04 & 18.01 & 12.52
\end{tabular}

The Au4f percentage decrease indicates coating of the Au surface with the ELR-based thin film since the peak intensity undergoes an exponential attenuation with the electron path length travelled. In additions, the similar Au4f percentage obtained for functionalized surfaces (Table 1) suggests ELR coatings with similar thicknesses. 


\subsection{Scanning Electron Microscopy (SEM)}

SEM morphological analysis allowed high-resolution imaging of the gold surfaces before (Figure 3a) and after functionalization with the three recombinamers used in this work (Figures 3b-d). Au clusters, formed as a result of sputtering deposition of gold on the glass surface, can clearly be seen in Figure 3a. The size of these clusters depends on the deposition rate and the different probability of gold atom capture on the bare glass substrate. [41, 42] An initial study of the coating effectiveness shows a uniform coverage of the surfaces (see Figures 3b-d) as the gold clusters have been smoothed with the applied coating.

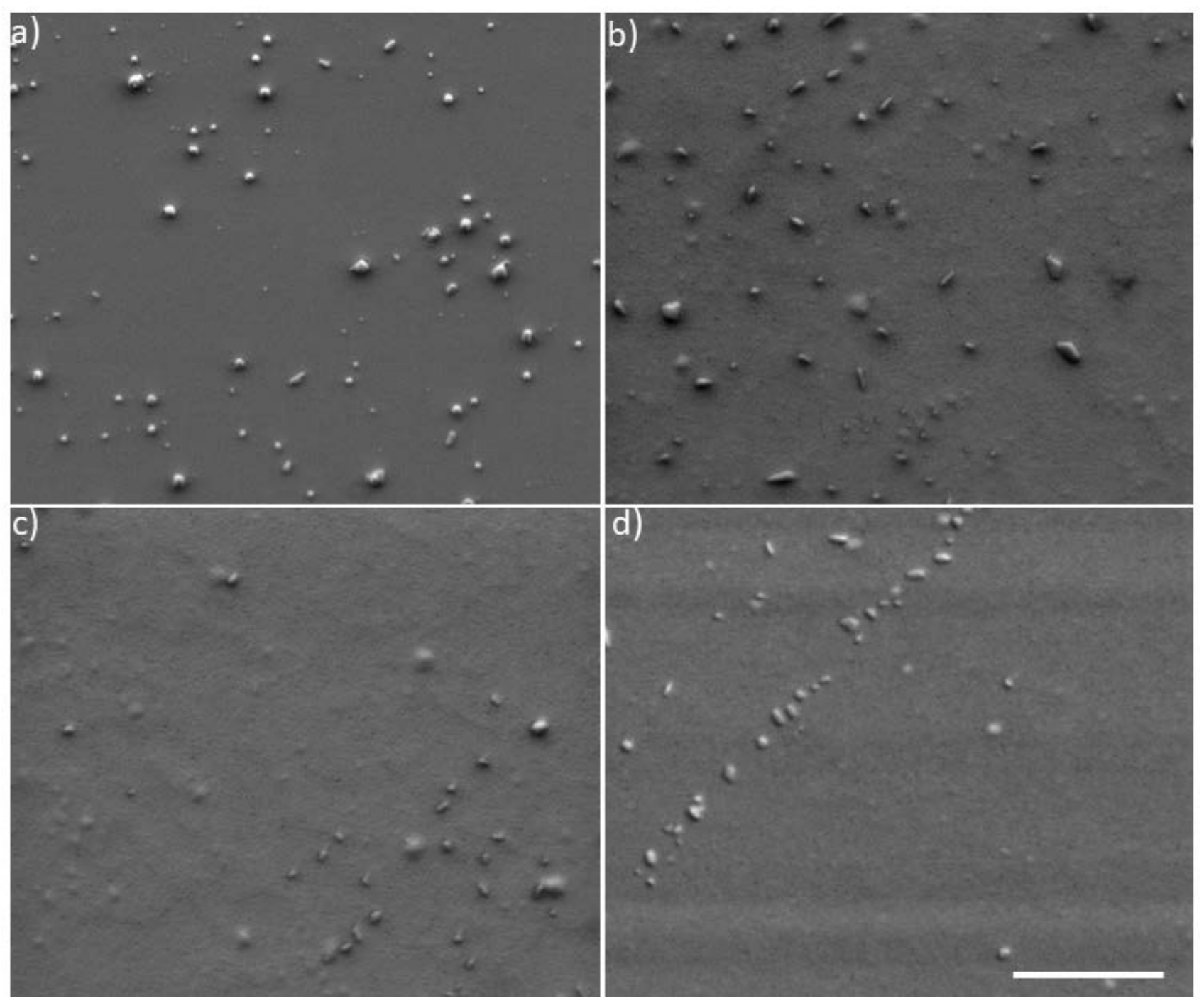

Figure 3. SEM micrographs of: a) non-functionalized gold surfaces (control); b) a gold surface functionalized with CC-RGD; c) a gold surface functionalized with CCC-REDV; d) a gold surface functionalized with CCC-EI. Scale bar: $4 \mu \mathrm{m}$. 
Ablated strip micrographs (Fig. 4a-b) show an absence of damage and marks on the surface. This morphology agrees with that of the control surface (Figure 4c), which consists of Au clusters formed during the Au sputtering deposition process.
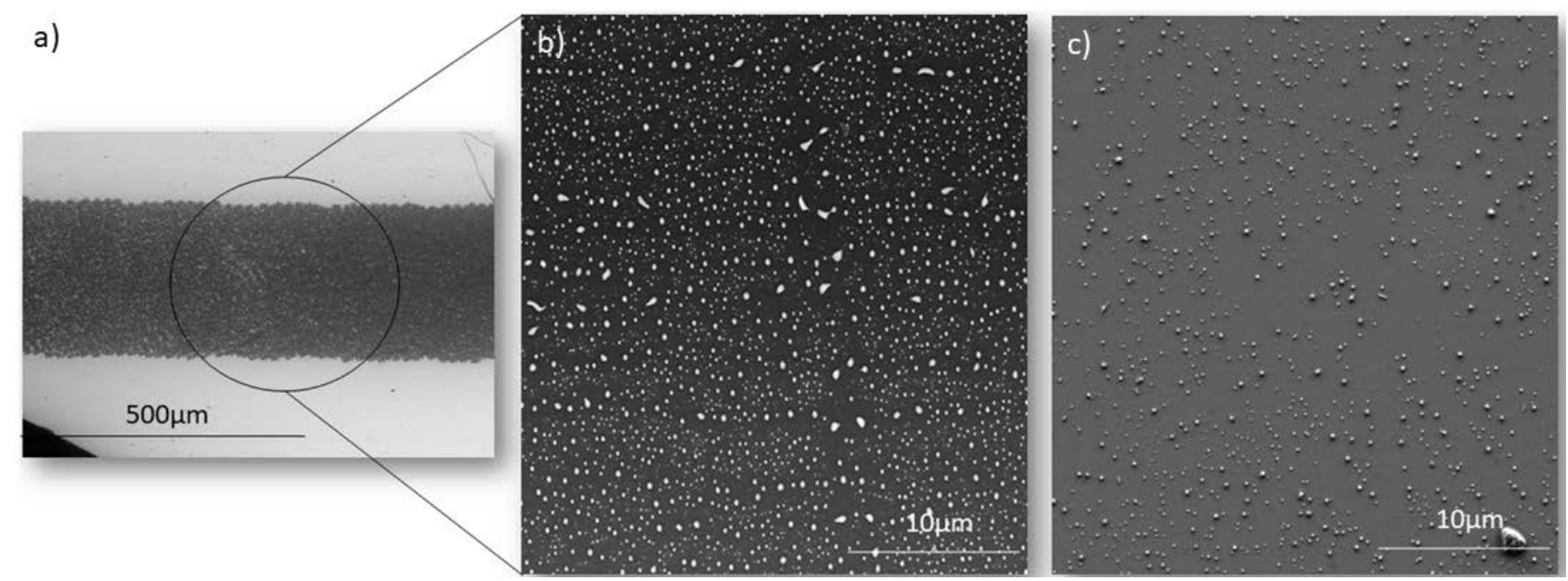

Figure 4. a) Strip ablated with MALDI TOF/TOF UltrafleXtrem instrument, b) magnified image of the ablated strip, and c) non-functionalized (control) surface.

\subsection{Atomic Force Microscopy (AFM)}

The morphology and roughness of the surfaces were assessed by AFM, comparing the functionalized surfaces with the non-functionalized one (Figure 5). Clear changes in surface topography can be seen after functionalization. In agreement with the morphology observed in the SEM micrographs, although gold clusters can clearly be seen in the non-functionalized sample, the coating process gives rise to a granular surface where these clusters have been covered. The average RMS roughness of the bare gold surface $(4.79 \pm 0.2 \mathrm{~nm})$ is significantly reduced when the polymer is coated onto the whole Au surface (REDV: $0.40 \pm 0.02 \mathrm{~nm}$; RGD: $0.46 \pm 0.07 \mathrm{~nm}$; and EI: $0.81 \pm 0.1 \mathrm{~nm}$ ). As can be seen, a low average RMS roughness value is found (lower than $1 \mathrm{~nm}$ for every polymer coating). Moreover, the low standard deviation indicates a uniform coating of the surface.

The roughness of the strips was analyzed by AFM. After functionalization, the RMS roughness is $0.9 \pm 0.2 \mathrm{~nm}$, which is lower than the roughness for bare gold, thus meaning that the mixture of recombinamers has been deposited onto the strips (Fig. 5e). 

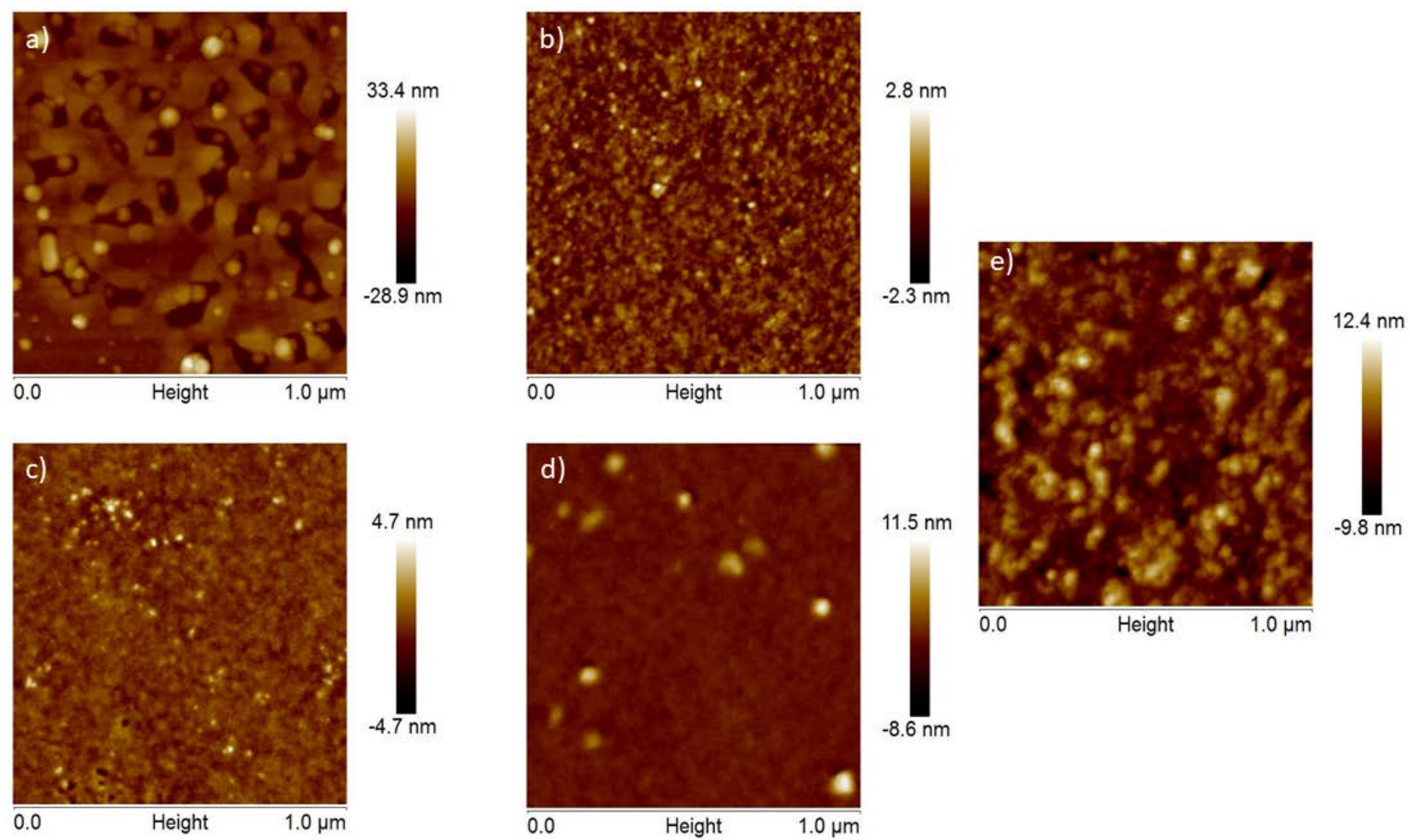

Figure 5: Different nano-topographies visualized using AFM technique: a) the-non-functionalized (control) surface, b) CCC-REDV, c) CC-RGD, d) CCC-EI and e) 75\% CCC-REDV/25\% CC-RGD. Scanned area: $1 \mathrm{x} 1 \mu \mathrm{m}^{2}$.

\subsection{Quartz Crystal Microbalance with Dissipation}

To complete the physical characterization of the surfaces functionalized with the ELRs, quartz crystal microbalance (QCM-D) was used as it allows to determine the thickness as well as layer's softness of a film deposited in a liquid environment.

Figure 6 shows the typical raw data from the QCM-D measurements. The simultaneously measured changes in frequency (normalized to the corresponding overtone, $n$ ), $\Delta \mathrm{f}_{\mathrm{n}} / \mathrm{n}$ (Figure 6 a), and in energy dissipation, $\Delta \mathrm{D}_{\mathrm{n}}$ (Figure 6b), obtained at $\mathrm{n}=3(15 \mathrm{MHz}), \mathrm{n}=5(25 \mathrm{MHz})$, and $\mathrm{n}=7(35 \mathrm{MHz})$ are displayed as a function of time. Although measurements were carried out up to the 13th overtone (65 MHz) under our experimental conditions, only the first three harmonics are shown for clarity. The frequency change at the fundamental frequency is not generally analyzed since this is affected by the 
flow of the bulk solution and is prone to more pronounced instabilities than the overtones.[43] [44] [45] Thus, only overtones are used for further quantitative analysis.

Three events can be distinguished in the transient evolution: i) flow of pure PBS to establish the baseline, ii) flow of the polymer solution, followed by iii) a final pure buffer rinse.

At this point we will focus our attention on the results obtained for pure CCC-REDV and pure CCRGD (Fig.6). Upon exposure to the polymer solution, the decrease in frequency indicates adsorption on the side of the sensor. In addition, whereas relatively saturated values were obtained for CCCREDV at the end of the polymer solution flow time (1200 sec.), no saturation was observed for CCRGD. A higher frequency change was observed for CCC-REDV (around -53 Hz; n = 3) than for CCRGD (around $-35 \mathrm{~Hz} ; \mathrm{n}=3$ ). This mass uptake is accompanied by a strong increase in dissipation for both CCC-REDV and CC-RGD (up to $10 \times 10^{-6} ; \mathrm{n}=3,7.8 \times 10^{-6} ; \mathrm{n}=3$, respectively).

The final event in the QCM-D experiment corresponds to rinsing with pure PBS solution to stabilize and eliminate any material accumulated on the surface. (1300 sec.) Thus, whereas fairly small variations are found in the frequency changes, a significant dissipation reduction for both ELRs is observed (at saturation, $5 \times 10^{-6} ; \mathrm{n}=3$ and $3.2 \times 10^{-6} ; \mathrm{n}=3$ for CCC-REDV and CC-RGD, respectively). These experimental results suggest that the thin film stabilization leads to a stiffer and most likely to a more compact film than prior to the PBS rinse. 

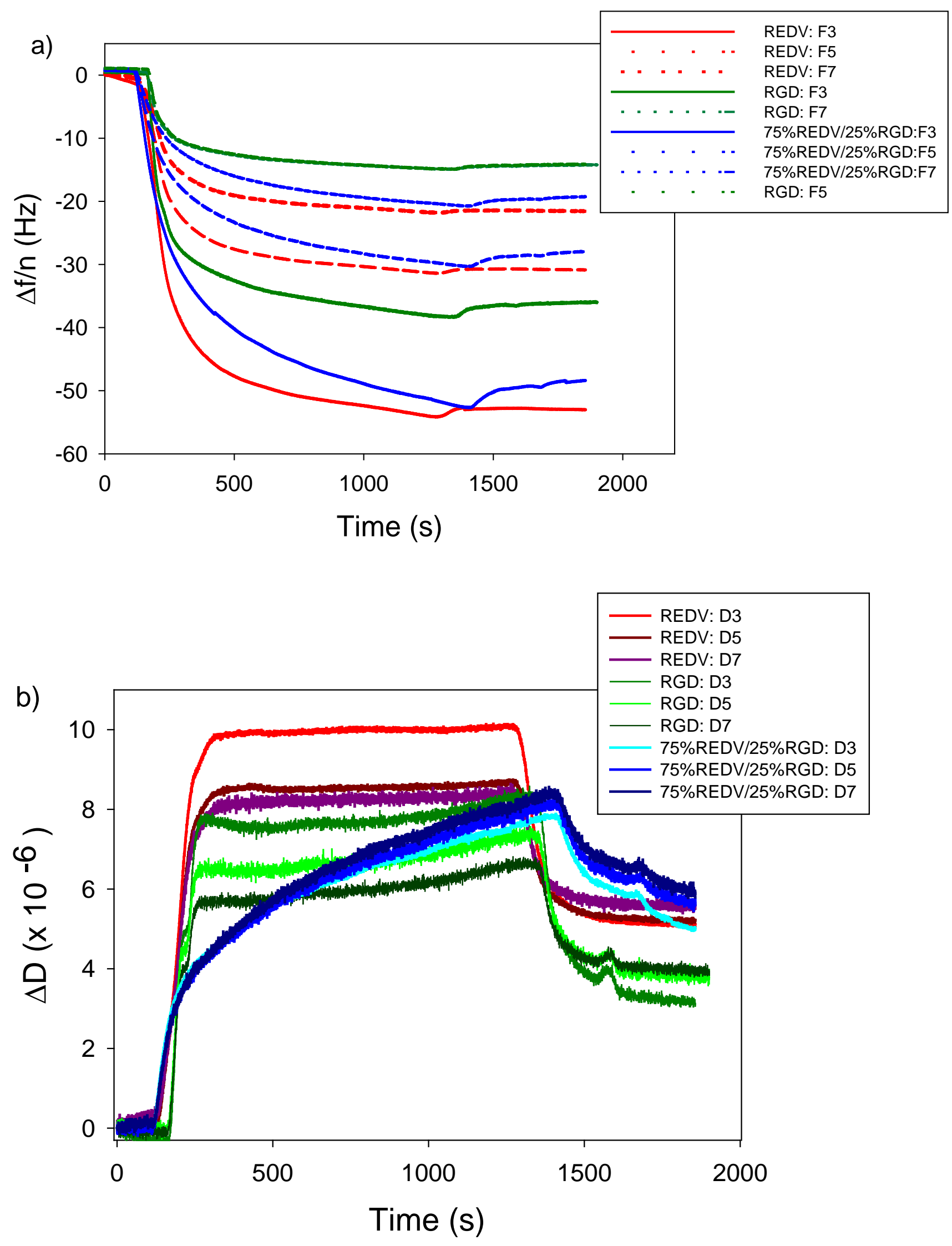
Figure 6: Normalized frequency (a) and energy dissipation shifts (b) measured at the third $(\mathrm{n}=3)$, fifth $(n=5)$, and seventh $(n=7)$ overtones for CCC-REDV, CC-RGD and the mixture 75\% REDV/25\% RGD. Three events are distinguished: first, pure buffer (PBS) solution for 3 min.; second, exposure to ELR solution for $20 \mathrm{~min}$., and finally, pure buffer solution again for $10 \mathrm{~min}$.

The frequency dependence observed for the three overtones in Figure 6a, and the high energy dissipation, indicate that the simple Sauerbrey model [51] is not valid and therefore that a viscoelastic model is required. In this case, a Voight viscoelastic model [52-53] based on a single layer was used. The adsorbed film is represented by a lateral homogeneous film with uniform thickness and density which, in our case, was estimated at $1.1 \mathrm{~g} / \mathrm{cm}^{3}$ (corresponding to a hydrated protein).[46] This film is situated between the sensor surface and on its other side is in contact with a semi-infinite bulk solution (in our case, PBS) that was assumed to be Newtonian, with a viscosity and density of $0.92 \mathrm{mPa} . \mathrm{s}$ and $1.005 \mathrm{~g} / \mathrm{cm}^{3}$, respectively.

No significant impact of surface roughness on the QCM experimental values is expected since, given the average RMS roughness obtained by AFM (less than $1 \mathrm{~nm}$ for each polymer coating), the coated QCM sensor can be regarded as flat.[47] Moreover, within the resolution of the AFM images (Fig. 5) the thin film can be considered to be laterally homogeneous.

Subsequent characterization of the adsorbed thin film was based on the QCM-raw data corresponding to the polymer solution flowing for $20 \mathrm{~min}$. Several sets of raw experimental data and the corresponding fitted curves can be found in the Supporting Information (Fig. S7).

At the end of the second event (1700 sec.), the best fit for CCC-REDV was obtained for a film represented by a Voight thickness of $32.7 \pm 3.0 \mathrm{~nm}$, an elastic modulus of $219.2 \pm 8.0 \mathrm{kPa}$, and a viscosity of $3.758 \pm 0.700 \mathrm{mPa}$.s. The corresponding parameters for CC-RGD were $25.9 \pm 2.6 \mathrm{~nm}$, $101.3 \pm 12.1 \mathrm{kPa}$, and $2.386 \pm 0.354 \mathrm{mPa}$.s, respectively. Similarly, under steady-state conditions in the final PBS rinse (third event), the CCC-REDV film was represented by the following set of parameters: $29.1 \pm 4.1 \mathrm{~nm}, 402.0 \pm 155.4 \mathrm{kPa}$, and $8.400 \pm 0.454 \mathrm{mPa} . \mathrm{s}$, respectively. Furthermore, a wet area (Voight) mass of $3598 \pm 503 \mathrm{ng} / \mathrm{cm}^{2}$ was obtained. The corresponding values for CC-RGD were $24.0 \pm 3.6 \mathrm{~nm}, 108.1 \pm 24.0 \mathrm{kPa}, 3.155 \pm 0.887 \mathrm{mPa} . \mathrm{s}$, and $2581 \pm 487 \mathrm{ng} / \mathrm{cm}^{2}$, respectively. 
These parameters suggest that the CCC-REDV thin film is slightly thicker than its CC-RGD counterpart and has a better mechanical performance (i.e., higher storage modulus and viscosity). With respect to the Voight mass, it should be pointed out that the rheological parameters are measured in the $\mathrm{MHz}$ frequency range, whereas rheological characterization is usually accomplished in the $\mathrm{Hz}-\mathrm{kHz}$ range. It should also be noted that, during QCM measurements, liquid or solvent molecules may couple as an additional mass via direct hydration, viscous drag, or entrapment in cavities in the adsorbed film. [48] The comparison of these results confirms the stabilization of the film (namely a stiffer and more compact film) after the PBS rinse for both ELRs since the elastic modulus and viscosity increase, and that the thickness decreases.

\subsection{Selective cell adhesion on ELR-bio functionalized model gold surfaces}

The next step in this work consisted in determining the optimum mixture of CC-RGD and CCCREDV in order to obtain selective adhesion of endothelial cells. A co-culture of HUVEC and HFF1 cells was used to that end. Adhesion was carried out under serum-free conditions in order to avoid the interference of soluble extracellular matrix (ECM) proteins found in the serum with the observed behavior.

To achieve this goal, whole gold surfaces were functionalized with ELR mixtures comprising varying percentages of each ELR: a) 100\% CC-RGD, b) 25\% CCC-REDV/75\% CC-RGD, c) $50 \%$ CCCREDV/50\% CC-RGD, d) 75\% CCC-REDV/25\% CC-RGD, and e) 100\% CCC-REDV. A surface functionalized with CCC-EI, which does not contain any bioactive sequence, was used as a negative control. In Figure 7 the adhesion of HUVEC (green) and HFF1 (red) on each functionalized surface with different percentages of the recombinamer mixture can be seen from top to bottom: phase contrast (A1-A6), FITC channel (B1-B6, only the HUVEC cells are visualized), G-2A channel (C1-C6, for HFF1 visualization) and, finally, all channels merged (D1-D6).

For HUVEC cells, there was no significant difference in adhesion on surfaces functionalized with $0 \%$, 25\% and 100\% CCC-REDV, although a relatively minor increase in adhesion of these cells was observed on surfaces functionalized with 50\% CCC-REDV. In contrast, a significant increase in the adhesion of HUVECs on surfaces functionalized with 75\% CCC-REDV and 25\% CC-RGD was 
observed. The quantity of cells adhered onto one square centimeter of various surfaces can be seen in Figure 8.

It can clearly be seen that the presence of the REDV sequence minimizes HFF1 adhesion.

Thus, as the percentage of CCC-REDV recombinamer increases, the number of HFF1 cells adhered decreases considerably, thereby highlighting the significance of the REDV ligand for selective adhesion of endothelial cells on model gold surfaces. The presence of this motif provides an unfavorable environment for this cell type. In light of the above, we decided to use the mixture $75 \%$ CCC-REDV/25\% CC-RGD as the optimal mixture for our spatially selective cell cultures given the enhanced adhesion of endothelial cells.

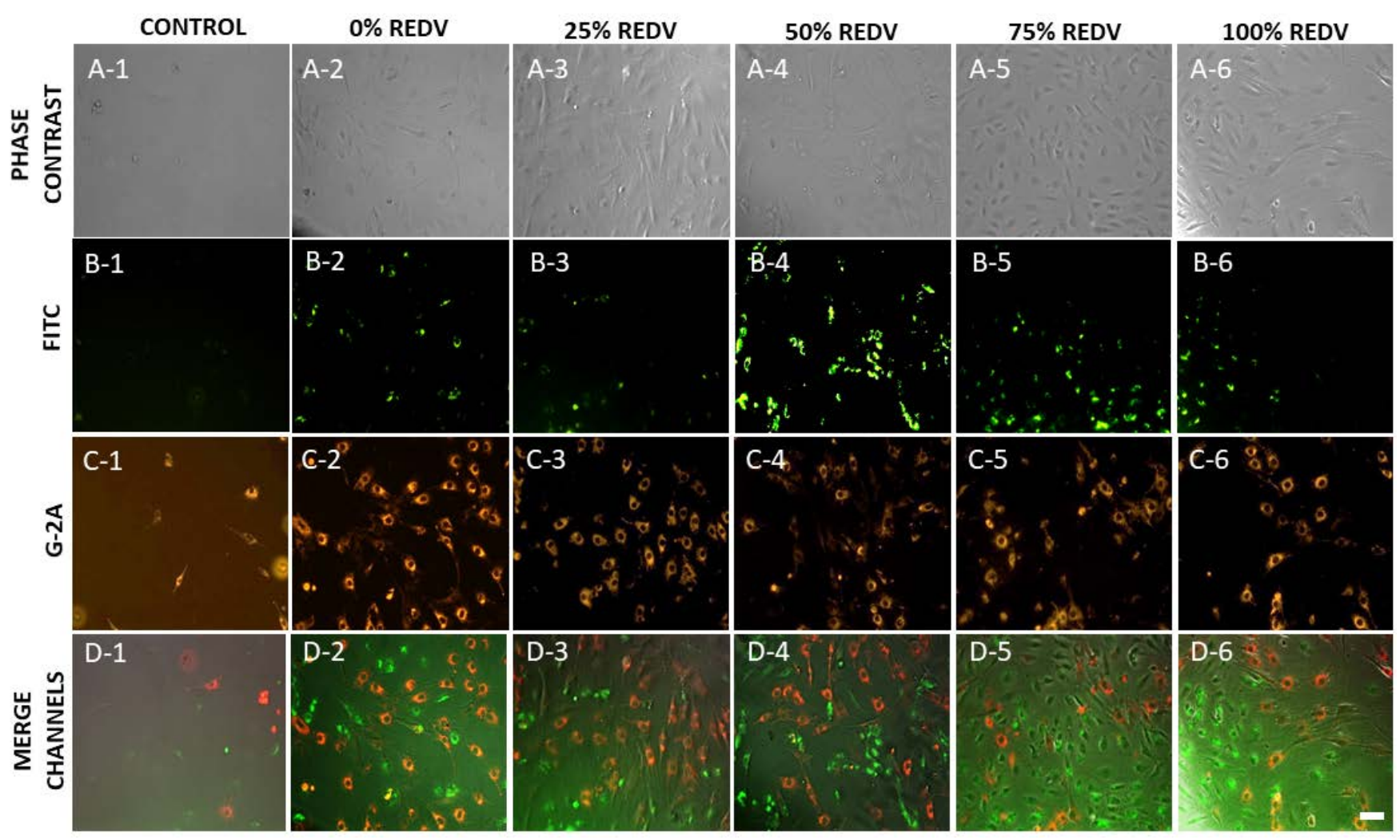


Figure 7. HUVEC (membrane labeled with PKH67 Green Fluorescent Cell Linker Kit) and HFF1 (membrane labeled with PKH26 Red Fluorescent Cell Linker Kit) adhered to surfaces functionalized with 0\% CCC-REDV, 25\% CCC-REDV, 50\% CCC-REDV, 75\% CCC-REDV and 100\% CCC-REDV, respectively. From top to bottom: image A shows the phase contrast for each percentage functionalization; image B the FITC channel to visualize HUVEC cells; image $C$ the G2A channel to visualize HFF1, and finally, all channels are merged in image D. Scale bar: $50 \mu \mathrm{m}$.

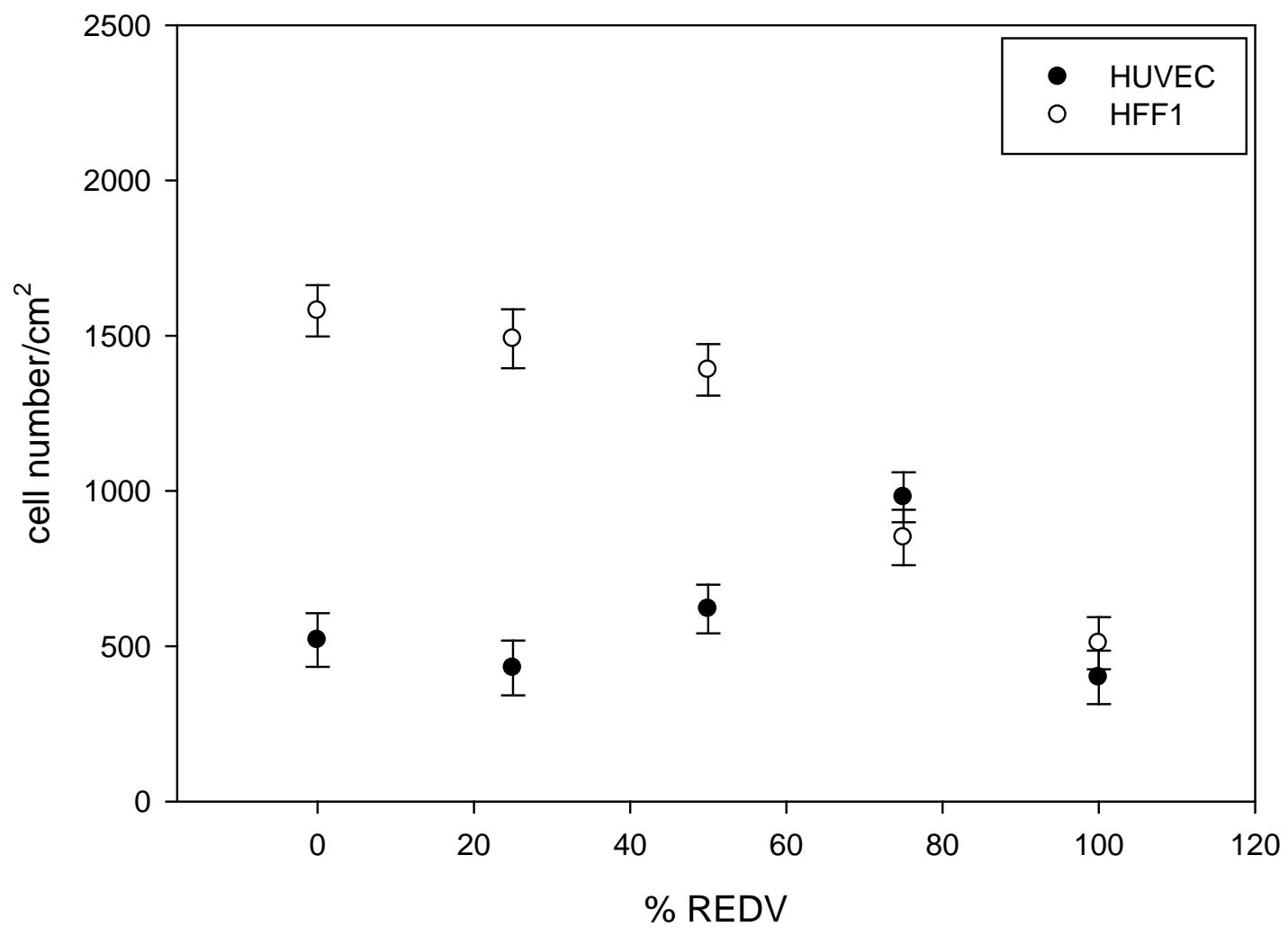

Figure 8: Number of HUVEC (black) and HFF1 cells (white) adhered onto surfaces functionalized with different percentages of ELR (100\% CC-RGD, 25\% CCC-REDV/75\% CC-RGD, 50\% CCCREDV/50\% CC-RGD, 75\% CCC-REDV/25\% CC-RGD and 100\% CCC-REDV).

\subsection{Spatial control of endothelial cell adhesion}

Once the optimal percentage for selective adhesion of endothelial cells and the protocol for cleaning gold strips by laser ablation had been established, the next step was the selective functionalization of these strips. Thus, the strips were functionalized with the mixture of 75\% CCC-REDV and 25\% CCRGD. This functionalization only takes place on those strips where a bare gold surface is available as the rest of the surface has been previously functionalized with the CC-RGD recombinamer. 
These surfaces were submitted to a co-culture of endothelial and HFF1 cells for 4 hours in order to demonstrate a maximal selective adhesion onto the strips. Cells were counted using ImageJ software. Interestingly, we observed a significant increase in the adhesion of endothelial cells on the strips functionalized with 75\% CCC-REDV/25\% CC-RGD when compared with the other areas functionalized with 100\% CC-RGD. Indeed, Figure 9 clearly shows that the number of endothelial cells (green) (Fig. 9 C) adhered onto the surfaces is higher than that for HFF1 (red) (Fig. 9 B). Specifically, the number of endothelial cells adhered onto the strips is $520 \pm 35$, while the value for HFF1 cells is $175 \pm 20$. A noticeable spatial control of HUVEC adhesion on the strip is therefore demonstrated.

PHASE CONTRAST A)

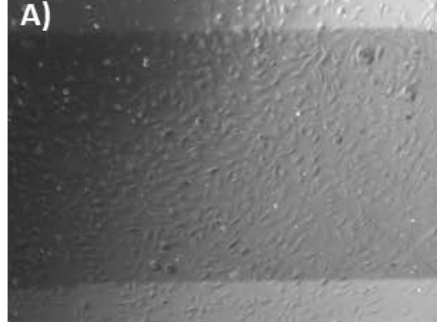

\section{G-2A}

FITC

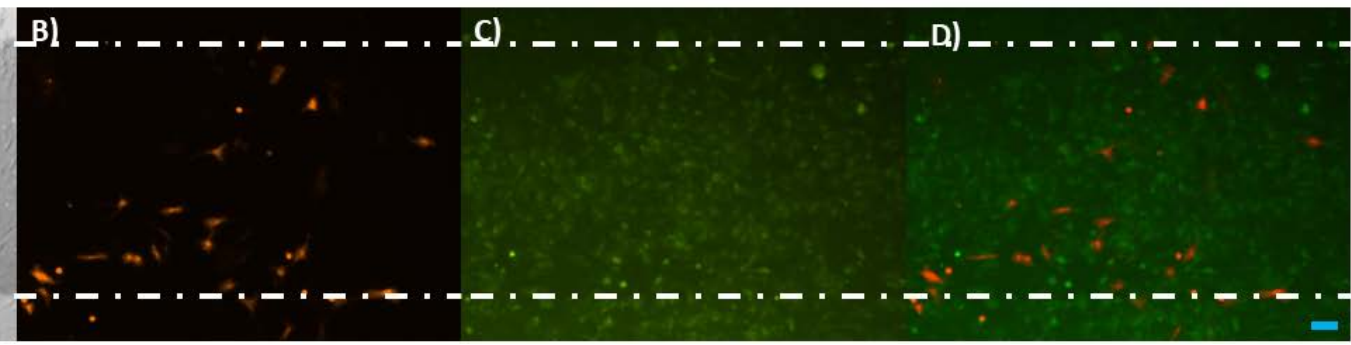

Figure 9. From left to right: image A shows the phase contrast of the strip; image B the G-2A channel to visualize HFF1 adhered onto the strip; image C the FITC channel to visualize HUVEC cells, and finally all channels have been merged in image D. Scale bar: $50 \mu \mathrm{m}$. 


\section{Discussion}

REDV sequence plays a critical role in the adhesion of endothelial cells while exhibits better cell-type selectivity due to its receptor in their membrane which is only expressed in limited cell-lines. [49] [50] Although, this sequence is considered to be highly selective for endothelial cells, when present on its own it promotes only poor and weak adhesion despite its selectivity. [49] This validates the results obtained to date, namely that the presence of the RGD sequence in the mixture used to functionalize the surfaces is necessary to facilitate the adhesion of endothelial cells.

Overall, we have demonstrated that the REDV sequence is a selective bioactive domain for endothelial cells by increasing their adhesion to a model gold surface. This selectivity towards endothelial cells in the presence of the REDV sequence further underlies the crucial bioactivity and, more importantly, selectivity provided by this ligand in cell adhesion and allowed us to demonstrate that adhesion of endothelial cells is markedly enhanced on those surfaces functionalized with 75\% CCC-REDV and 25\% CC-RGD. The adhesion ratio of endothelial cells was significantly higher than for HFF1 cells, thus indicating a selective adhesion with respect to endothelial cells.

In addition of cell adhesion sequences such as REDV and RGD, the ELRs contain cysteines at the amino-terminal region of their backbone as these are essential for grafting onto gold and avoid the use of intermediates such as alkane thiols. As reported in literature [51] there are considerable limitation on using chemical approaches like biocompatibility, applicability to only a limited number of surfaces, high costs, low yield and stability that are overcome with the use of genetically engineered recombinamers. Apart from potential receptor-mediated interactions, physical and chemical factors, including surface chemistry, topography and hydrophilicity, are key to the long-term viability and proliferation of cells.

Protein grafting on model gold surfaces was assessed using several techniques, such as contact angle, XPS, SEM, AFM, and QCM-D. The contact angle values highlighted an increase in hydrophobicity with no significant differences in the functionalized surfaces. As can be seen from the literature, this results confirm that clean gold is hydrophilic and deposition of a recombinamer monolayer renders it hydrophobic.[52] 
The chemical composition of the surfaces was studied by XPS, which showed a clear variation in atomic levels at the surface. Both the significant increase in the percentage of C1s and N1s and the marked reduction in the intensity of Au in comparison with the non-functionalized surface indicate the effective coating of gold surface with a similar thickness for every functionalized surface on which covalent Au-S bonding occurs. The Au4f $f_{7 / 2}$ peak typically appears at around $83-84 \mathrm{eV}$ for $\mathrm{Au}(0)$ in the XPS spectrum. [53] [54] [55] [56] When Au (I) thiolates are formed, a positive shift in binding energy of about 0.2-1.0 eV has been reported. [54] In accordance with this, our XPS spectra (see Fig. S6 in Supporting Information) show a positive shift of around $0.3 \mathrm{eV}$, thus indicating Au-S covalent bonding in the thin ELR film.

Following with the characterization, the morphology and nano-topography of the surfaces were studied in more detail by SEM microscopy and AFM, respectively, thereby offering an initial insight into the grafting effectiveness and showing homogenous coverage of the surface topography. The RMS roughness decrease when gold surfaces are functionalized with the recombinamers having similar values lower than $1 \mathrm{~nm}$.

Numerical fitting of the QCM-D experimental data to a Voight viscoelastic model indicated a stabilization of the adsorbed film after the PBS rinse. QCM characterization of the thin film based on the mixture of both ELRs was then carried out. The QCM-D measurements for the pure polymers and their mixture (75\% REDV-25\% RGD) are shown in Figure 6. For the polymer mixture, no saturation is achieved in the 20 min during which the polymer solution is flowing. Moreover, given the frequency shift (Figure 6a) at the end of the measurements (after the PBS rinse), the frequency change tends to be similar to that for $100 \%$ REDV, although the transient evolution is clearly different. As for dissipation (Figure 6b), a significant difference (with respect to pure solutions) is again found during polymer solution flow, with no saturation being observed at the end of the experimental time (20 min.). Nevertheless, at the end of the buffer rinse, a similar dissipation is observed for both $100 \%$ REDV and the polymer mixture. These experimental results are consistent with the higher REDV percentage present in the mixture (75\%). Under steady-state conditions in the final PBS rinse (third event), the 75\% CCC-REDV/25\% CC-RGD thin film exhibits the following parameters: $31.5 \pm 1.3 \mathrm{~nm}, 171 \pm 38$ $\mathrm{kPa}, 3.183 \pm 0.601 \mathrm{mPa} . \mathrm{s}$, and $3550 \pm 228 \mathrm{ng} / \mathrm{cm}^{2}$. These parameters are closer to those for $100 \%$ 
REDV than for $100 \%$ RGD, although the elastic modulus and viscosity of this film are lower. The QCM-D measurements taken during the 6 hours of polymer solution flow (see Fig. S8 in Supporting Information) indicate a saturated thickness and areal mass of $38.8 \pm 3.1 \mathrm{~nm}$ and of $4300 \pm 483 \mathrm{ng} / \mathrm{cm}^{2}$, and $44 \pm 3.4 \mathrm{~nm}$ and $4850 \pm 535 \mathrm{ng} / \mathrm{cm}^{2}$ are obtained, for pure RGD and the polymer mixture, respectively, after the PBS rinse. As such, no significant differences in thickness are observed between the mixture-functionalized strip and the rest of the RGD-functionalized surface. Overall these results therefore suggest that the ELRs are grafted onto the surfaces giving rise to functionalized surfaces.

Specific regions of the surfaces (strips) were cleaned by laser ablation without altering their bulk properties and were subsequently characterized by SEM and AFM confirming that gold had not been removed. A comparison of the SEM micrographs for the as-received gold surface and ablated strips showed no major differences. Consequently, these strips were functionalized with the optimized mixture (75\% CCC-REDV-25\% CC-RGD). No topographic differences has been found among the functionalized surfaces and the re-functionalized strips. The results demonstrated that spatially controlled selective cell adhesion was bio-specific on strips functionalized with the optimized mixture of recombinamers, which showed a better ability to promote endothelial cell adhesion due to the presence of the REDV motif. [22] [57]

Since no significant differences in topography, hydrophobicity and mechanical properties are found in each functionalized surface (including strips), the cell selectivity and spatially controlled cell adhesion is related to the presence of the bioactive sequences existing into the backbone of the ELRs.

This study could be applied in different fields, such as drug discovery, biomedical engineering, and intercellular applications, as well as to the study of fundamental cell biology and the production of cell-based biosensors and diagnostic devices. [58] In addition, this novel system provides a reliable and accessible tool with sufficient flexibility to address a wide range of biological and engineering problems that require control over the spatial and temporal organization of cells. [59] 


\section{Conclusions}

Model gold surfaces functionalized with a specific adhesion motif have proven to be a valuable tool to achieve spatially controlled selective cell adhesion. To that end, a universal cell attachment sequence (RGD) and an endothelial cell specific adhesion sequence (REDV) were included in two ELRs to regulate the adhesive and migratory behavior of cells. Using a co-cultures of HFF1 and HUVEC cells, a selective adhesion of endothelial cells was obtained on surfaces grafted with a mixture of 75\% CCC-REDV and 25\% CC-RGD. Strips cleaned by laser ablation were subsequently functionalized by a mixture of the ELRs (75\% CCC-REDV - 25\% CC-RGD). Each functionalized surface (including strips) has similar topography, hydrophobicity and mechanical properties according to the combination of general experimental techniques applied herein (contact angle, SEM, AFM, XPS and QCM-D). In fact, strips functionalized with the optimal mixture (75\% CCCREDV and 25\% CC-RGD) demonstrate a spatially controlled adhesion of endothelial cells due to the presence of the bioactive RGD and REDV domains. The ability to spatially control cell adhesion and multicellular organization is critical to many biomedical and tissue-engineering applications and has become increasingly important for the development of cellular biosensor technology and tissue engineering applications.

\section{Acknowledgments}

The authors are grateful for the funding from the European Commission (NMP-2014-646075, MSCA-ITN-2014-642687), MINECO of the Spanish Government (PCIN-2015-010, MAT201568901-R, MAT2016-78903-R and MAT2016-79435-R), Junta de Castilla y León (VA015U16) and Centro en Red de Medicina Regenerativa y Terapia Celular de Castilla y León. 


\section{References}

[1] Ku SH, Lee JS, Park CB. Spatial control of cell adhesion and patterning through musselinspired surface modification by polydopamine. Langmuir 2010;26:15104-8.

[2] Shin H, Jo S, Mikos AG. Biomimetic materials for tissue engineering. Biomaterials 2003;24:4353-64.

[3] von der Mark K, Park J, Bauer S, Schmuki P. Nanoscale engineering of biomimetic surfaces: cues from the extracellular matrix. Cell and tissue research 2010;339:131.

[4] Frey BL, Jordan CE, Kornguth S, Corn RM. Control of the specific adsorption of proteins onto gold surfaces with poly (l-lysine) monolayers. Analytical Chemistry 1995;67:4452-7. [5] Sawyer A, Hennessy K, Bellis S. Regulation of mesenchymal stem cell attachment and spreading on hydroxyapatite by RGD peptides and adsorbed serum proteins. Biomaterials 2005;26:1467-75.

[6] Wirkner M, Weis S, San Miguel V, Álvarez M, Gropeanu RA, Salierno M, et al. Photoactivatable caged cyclic RGD peptide for triggering integrin binding and cell adhesion to surfaces. ChemBioChem 2011;12:2623-9.

[7] Sharon J, Puleo D. Immobilization of glycoproteins, such as VEGF, on biodegradable substrates. Acta biomaterialia 2008;4:1016-23.

[8] Pohl TL, Boergermann JH, Schwaerzer GK, Knaus P, Cavalcanti-Adam EA. Surface immobilization of bone morphogenetic protein 2 via a self-assembled monolayer formation induces cell differentiation. Acta biomaterialia 2012;8:772-80.

[9] Ostuni E, Yan L, Whitesides GM. The interaction of proteins and cells with selfassembled monolayers of alkanethiolates on gold and silver. Colloids and Surfaces B: Biointerfaces 1999;15:3-30.

[10] Abad JM, Pita M, Fernández VM. Immobilization of proteins on gold surfaces. Immobilization of Enzymes and Cells 2006:229-38.

[11] Anselme K, Ploux L, Ponche A. Cell/material interfaces: influence of surface chemistry and surface topography on cell adhesion. Journal of Adhesion Science and Technology 2010;24:831-52.

[12] Rodríguez-Cabello JC, Martín L, Girotti A, García-Arévalo C, Arias FJ, Alonso M. Emerging applications of multifunctional elastin-like recombinamers. Nanomedicine 2011;6:111-22.

[13] Rodríguez-Cabello JC, Martín L, Alonso M, Arias FJ, Testera AM. “Recombinamers” as advanced materials for the post-oil age. Polymer 2009;50:5159-69.

[14] Machado R, Ribeiro AJ, Padrão J, Silva D, Nobre A, Teixeira J, et al. Exploiting the sequence of naturally occurring elastin: construction, production and characterization of a recombinant thermoplastic protein-based polymer. Journal of Nano Research: Trans Tech Publ; 2009. p. 133-45.

[15] Girotti A, Fernández - Colino A, López IM, Rodríguez - Cabello JC, Arias FJ. Elastin like recombinamers: Biosynthetic strategies and biotechnological applications. Biotechnology journal 2011;6:1174-86.

[16] Ibáñez - Fonseca A, Ramos TL, Torre IG, Sánchez - Abarca LI, Muntión S, Arias FJ, et al. Biocompatibility of two model elastin - like recombinamer - based hydrogels formed through physical or chemical crosslinking for various applications in tissue engineering and regenerative medicine. Journal of Tissue Engineering and Regenerative Medicine 2017. [17] Urry DW. Molecular machines: how motion and other functions of living organisms can result from reversible chemical changes. Angewandte Chemie International Edition 1993;32:819-41.

[18] Urry DW, Gowda D, Parker TM, Luan CH, Reid MC, Harris CM, et al. Hydrophobicity scale for proteins based on inverse temperature transitions. Biopolymers 1992;32:1243-50. 
[19] Garcia-Arevalo C, Pierna M, Girotti A, Arias FJ, Rodriguez-Cabello JC. A comparative study of cell behavior on different energetic and bioactive polymeric surfaces made from elastin-like recombinamers. Soft Matter 2012;8:3239-49.

[20] Mrksich M. A surface chemistry approach to studying cell adhesion. Chemical Society Reviews 2000;29:267-73.

[21] Urry D, Shaw R, Prasad K. Polypentapeptide of elastin: temperature dependence of ellipticity and correlation with elastomeric force. Biochemical and biophysical research communications 1985;130:50-7.

[22] Humphries MJ, Akiyama SK, Komoriya A, Olden K, Yamada KM. Identification of an alternatively spliced site in human plasma fibronectin that mediates cell type-specific adhesion. The Journal of Cell Biology 1986;103:2637-47.

[23] Panitch A, Yamaoka T, Fournier MJ, Mason TL, Tirrell DA. Design and biosynthesis of elastin-like artificial extracellular matrix proteins containing periodically spaced fibronectin CS5 domains. Macromolecules 1999;32:1701-3.

[24] Liu JC, Heilshorn SC, Tirrell DA. Comparative cell response to artificial extracellular matrix proteins containing the RGD and CS5 cell-binding domains. Biomacromolecules 2004;5:497-504.

[25] Ruoslahti E, Pierschbacher MD. New perspectives in cell adhesion: RGD and integrins. Science 1987;238:491-8.

[26] Ciofani G, Genchi GG, Liakos I, Athanassiou A, Mattoli V, Bandiera A. Human recombinant elastin-like protein coatings for muscle cell proliferation and differentiation. Acta biomaterialia 2013;9:5111-21.

[27] Wagner P, Hegner M, Kernen P, Zaugg F, Semenza G. Covalent immobilization of native biomolecules onto $\mathrm{Au}$ (111) via $\mathrm{N}$-hydroxysuccinimide ester functionalized selfassembled monolayers for scanning probe microscopy. Biophysical journal 1996;70:2052-66. [28] Demann ET, Stein PS, Haubenreich JE. Gold as an implant in medicine and dentistry. Journal of long-term effects of medical implants 2005;15.

[29] Qin D, Xia Y, Whitesides GM. Soft lithography for micro-and nanoscale patterning. Nature protocols 2010;5:491-502.

[30] Zhao X-M, Xia Y, Whitesides GM. Soft lithographic methods for nano-fabrication. Journal of Materials Chemistry 1997;7:1069-74.

[31] Sun S, Mendes P, Critchley K, Diegoli S, Hanwell M, Evans SD, et al. Fabrication of gold micro-and nanostructures by photolithographic exposure of thiol-stabilized gold nanoparticles. Nano letters 2006;6:345-50.

[32] Russo RE, Mao X, Liu H, Gonzalez J, Mao SS. Laser ablation in analytical chemistry-a review. Talanta 2002;57:425-51.

[33] Momma C, Nolte S, Chichkov BN, Alvensleben Fv, Tünnermann A. Precise laser ablation with ultrashort pulses. Applied surface science 1997;109:15-9.

[34] Liu X, Du D, Mourou G. Laser ablation and micromachining with ultrashort laser pulses. IEEE journal of quantum electronics 1997;33:1706-16.

[35] Momma C, Chichkov BN, Nolte S, von Alvensleben F, Tünnermann A, Welling H, et al. Short-pulse laser ablation of solid targets. Optics communications 1996;129:134-42.

[36] Yeh J. Laser ablation of polymers. Journal of Vacuum Science \& Technology A: Vacuum, Surfaces, and Films 1986;4:653-8.

[37] Chichkov BN, Momma C, Nolte S, Von Alvensleben F, Tünnermann A. Femtosecond, picosecond and nanosecond laser ablation of solids. Applied Physics A 1996;63:109-15.

[38] Vogel A, Venugopalan V. Mechanisms of pulsed laser ablation of biological tissues.

Chemical reviews 2003;103:577-644.

[39] Garrison BJ, Srinivasan R. Laser ablation of organic polymers: microscopic models for photochemical and thermal processes. Journal of Applied Physics 1985;57:2909-14. 
[40] Pensa E, Cortés E, Corthey Gn, Carro P, Vericat C, Fonticelli MH, et al. The chemistry of the sulfur-gold interface: in search of a unified model. Accounts of chemical research 2012;45:1183-92.

[41] Schwartzkopf M, Hinz A, Polonskyi O, Strunskus T, Löhrer FC, Körstgens V, et al. Role of sputter deposition rate in tailoring nanogranular gold structures on polymer surfaces. ACS applied materials \& interfaces 2017;9:5629-37.

[42] Malinský P, Slepička P, Hnatowicz V, Švorčík V. Early stages of growth of gold layers sputter deposited on glass and silicon substrates. Nanoscale research letters 2012;7:241.

[43] Mechler A, Praporski S, Atmuri K, Boland M, Separovic F, Martin LL. Specific and selective peptide-membrane interactions revealed using quartz crystal microbalance.

Biophysical Journal 2007;93:3907-16.

[44] Bailey CM, Kamaloo E, Waterman KL, Wang KF, Nagarajan R, Camesano TA. Size dependence of gold nanoparticle interactions with a supported lipid bilayer: A QCM-D study. Biophysical chemistry 2015;203:51-61.

[45] Nielsen SB, Otzen DE. Quartz Crystal Microbalances as Tools for Probing ProteinMembrane Interactions. Lipid-Protein Interactions: Methods and Protocols 2013:1-21.

[46] Malmström J, Agheli H, Kingshott P, Sutherland DS. Viscoelastic modeling of highly hydrated laminin layers at homogeneous and nanostructured surfaces: quantification of protein layer properties using QCM-D and SPR. Langmuir 2007;23:9760-8.

[47] Rechendorff K, Hovgaard MB, Foss M, Besenbacher F. Influence of surface roughness on quartz crystal microbalance measurements in liquids. Journal of applied physics 2007;101:114502.

[48] Höök F, Kasemo B, Nylander T, Fant C, Sott K, Elwing H. Variations in coupled water, viscoelastic properties, and film thickness of a Mefp-1 protein film during adsorption and cross-linking: a quartz crystal microbalance with dissipation monitoring, ellipsometry, and surface plasmon resonance study. Analytical chemistry 2001;73:5796-804.

[49] Heilshorn SC, DiZio KA, Welsh ER, Tirrell DA. Endothelial cell adhesion to the fibronectin CS5 domain in artificial extracellular matrix proteins. Biomaterials 2003;24:424552.

[50] Massia SP, Hubbell JA. Vascular endothelial cell adhesion and spreading promoted by the peptide REDV of the IIICS region of plasma fibronectin is mediated by integrin alpha 4 beta 1. Journal of Biological Chemistry 1992;267:14019-26.

[51] Kacar T, Zin MT, So C, Wilson B, Ma H, Gul - Karaguler N, et al. Directed self immobilization of alkaline phosphatase on micro - patterned substrates via genetically fused metal - binding peptide. Biotechnology and bioengineering 2009;103:696-705.

[52] Smith T. The hydrophilic nature of a clean gold surface. Journal of Colloid and Interface Science 1980;75:51-5.

[53] Crist BV. A review of XPS data-banks. XPS Reports 2007;1.

[54] Bourg M-C, Badia A, Lennox RB. Gold- sulfur bonding in 2D and 3D self-assembled monolayers: XPS characterization. The Journal of Physical Chemistry B 2000;104:6562-7.

[55] Yola ML, Atar N. A novel voltammetric sensor based on gold nanoparticles involved in p-aminothiophenol functionalized multi-walled carbon nanotubes: application to the simultaneous determination of quercetin and rutin. Electrochimica Acta 2014;119:24-31.

[56] Reimers JR, Ford MJ, Marcuccio SM, Ulstrup J, Hush NS. Competition of van der Waals and chemical forces on gold-sulfur surfaces and nanoparticles. Nature Reviews Chemistry 2017;1:0017.

[57] Faucheux N, Schweiss R, Lützow K, Werner C, Groth T. Self-assembled monolayers with different terminating groups as model substrates for cell adhesion studies. Biomaterials 2004;25:2721-30.

[58] Folch A, Toner M. Microengineering of cellular interactions. Annual review of biomedical engineering 2000;2:227-56. 
[59] Lu Y, Chen S. Micro and nano-fabrication of biodegradable polymers for drug delivery. Advanced drug delivery reviews 2004;56:1621-33. 